\title{
Case report of interest
}

\section{Compressive stenosis of the inferior vena cava due to localized ascites after living}

\section{donor liver transplantation}

Hirotaka Tokai, Susumu Eguchi, Akihiko Soyama, Masaaki Hidaka, Mitsuhisa

Takatsuki, Yujo Kawashita, Yoshitsugu Tajima, Takashi Kanematsu

Department of Surgery, Nagasaki University Graduate School of Biomedical Sciences,

1-7-1 Sakamoto, Nagasaki 852-8051, Japan.

Key words: Budd-Chiari, ascites, living donor liver transplantation, inferior vena cava

Short title: Stenosis of IVC due to ascites after LDLT

Offprint request to: S. Eguchi, M.D., Department of Surgery, Nagasaki University

Graduate School of Biomedical Sciences, 1-7-1 Sakamoto, Nagasaki 852-8051, Japan.

E-mail: sueguchi@net.nagasaki-u.ac.jp

Telephone: 81-95-849-7316 FAX: 81-95-849-7319 


\begin{abstract}
A 54-year-old woman was admitted to our hospital following the diagnosis of decompensated liver cirrhosis with hepatitis C. She underwent living donor liver transplantation using the left hepatic lobe with the middle hepatic vein donated by her husband. After the transplantation, the patient suffered from a large number of ascites with liver dysfunction. Computed tomography demonstrated stenosis of the supra-hepatic inferior vena cava (IVC) with focal collection of fluid. A second laparotomy was performed 19 days after transplantation. When the encapsulated localized ascites on both sides of the IVC was opened, the ascites were flushed away. Subsequently, the grafted liver was easily mobilized and was placed in the natural position without any tension, and the pressure gradient of the IVC was improved. Herein, we report a very rare case of compression stenosis of the IVC resulting in Budd-Chiari syndrome caused by localized encapsulated ascites.
\end{abstract}




\section{Introduction}

Budd-Chiari syndrome (BCS) is a clinical condition caused by obstruction of the hepatic vein and/or the inferior vena cava (IVC). ${ }^{1,2}$ BCS or hepatic venous outflow obstruction after liver transplantation is an uncommon complication, which unless recognized and corrected could lead to major morbidity. ${ }^{3-6}$ BCS after liver transplantation is mostly due to the small caliber of an anastomosis of the IVC, or due to a twist or kink at an anastomosis of the hepatic vein (HV) or IVC. Herein, we report a very rare case of BCS caused by localized encapsulated ascites.

\section{Case report}

A 54-year-old woman was admitted to our hospital following the diagnosis of decompensated liver cirrhosis with hepatitis C. She had previously undergone transcatheter arterial embolization for hepatocellular carcinoma. In addition, she suffered from several episodes of esophageal variceal bleeding that were treated by endoscopic variceal ligation.

Upon admission, the patient exhibited jaundice and ascites during physical examination. In chemistry, the Child-Pugh score was 10, indicating that she was grade C. She was considered to be a candidate for living donor liver transplantation, which was 
performed using the left hepatic lobe with the middle hepatic vein, donated by her husband. The graft weighed $420 \mathrm{~g}$, which accounted for $42 \%$ of the standard liver volume of the recipient. The common trunk of the left and middle HV of the graft was modified to enlargen the orifice, and was sutured to the common trunk of the left and middle HV of the recipient in an end-to-end fashion. The main trunk of the portal vein of the recipient was anastomosed to the left portal branch of the donor. After reperfusion of the graft, end-to-end anastomosis of the artery and duct-to-duct anastomosis of the bile duct was performed. After reconstruction, hepatopexy was performed by suturing both sides of the falciform ligaments. Intraoperative color Doppler ultrasonography confirmed excellent flow in the hepatic artery, the portal vein and the HV.

Two days after the transplantation, the patient developed a large amount of ascites with elevated serum levels of total bilirubin and transamylase, and delayed prothrombin time. Color Doppler ultrasonography demonstrated that the anastomosis of the HV was not narrowed, but that the outflow wave did not indicate a three-phased wave. Computed tomography demonstrated stenosis of the IVC and focal collection of fluid (Figure 1). Vena cavogram showed narrowing of IVC at the caudal side of the hepatic vein (Figure 2A), so that the pressure of the supra-hepatic IVC was 2-4 mmHg in contrast with 25-30 mmHg in the infra-hepatic IVC. While HV flow was not 
significantly congested (Figure 2B), the pressure in the HV was also high (24 mmHg).

Angioplasty was performed by balloon catheter, and the narrowing portion was easily expanded. However, the stenosis of the IVC could not be reduced. Hyperbilirubinemia and coagulopathy progressed, and drained ascites increased up to 5 liters per day. The tentative diagnosis was torsion of the IVC due to regeneration of the liver graft, and a second laparotomy was performed 19 days after transplantation with an IVC catheter in place to measure the pressure gradient over time. When the peritoneal cavity was entered, about $500 \mathrm{~mL}$ of serous ascites was collected. With dissection of the falciform ligament the hepatopexy was released, but the pressure gradient was not altered. When the area around the HV was exposed, localized ascites encapsulated by a thin membrane were observed on both sides of the IVC, which surrounded and applied pressure on the IVC. However, there were no other obvious causes, such as stenosis or torsion. When the capsule was opened bilaterally, the ascites were flushed away. Subsequently, the grafted liver was easily mobilized and was placed in the natural position without any tension, resulting in a gradient of the pressure of the infra-hepatic IVC pressure of 14-15 $\mathrm{cmH}_{2} \mathrm{O}$; virtually the same as that of the supra-hepatic IVC (13-14 mmHg). Intraoperative color Doppler ultrasonography showed 3-phased flow in the HV. After the second operation, the ascites were decreased (Figure 3) and the other 
biochemical parameters gradually returned to normal.

\section{Discussion}

Commonly, BCS or hepatic venous outflow obstruction can be caused by anatomical difficulties, such as fibrous changes of the vein, or by thrombosis, which can sometimes be related to rejection ${ }^{3-12}$. The pressure gradient across the site of stenosis during venography is useful to indicate whether the stenosis is haemodynamically sound, and that interventional therapy may be necessary. In our case, stenosis of the IVC from the caudal side was partially involved in the HV, since the pressure gradient between the SVC and hepatic vein was high. Taken together with the graft liver dysfunction before re-laparotomy, BCS due to the IVC stenosis caused by encapsulated ascites was considered the more appropriate pathological status in this patient. In addition, the deterioration of the graft function was not only due to ideal hepatic venous run off although it was patent, but also the result of systemic fluid imbalance as the result of IVC syndrome. Therefore, after the re-laparotomy with the result of decrease in IVC pressure gradient between supra and infra hepatic IVC, liver function was gradually ameliorated. In common cases, stenting or ballooning against the stenosis can provide a good outcome ${ }^{4,8,11}$, but our case was not suitable for intervention therapy. As a result, 
the stenosis of the IVC was found to be caused by localized ascites. Stenosis of the IVC or HV due to encapsulated localized ascites after liver transplantation has never been reported. However, the reason for the localization of the ascites remains unknown. It is suggested that encapsulated localized ascites can interrupt the flow of the IVC and that surgical removal of the ascites is effective. 


\section{References}

1. Janssen HL, Garcia-Pagan JC, Elias E, Mentha G, Hadengue A, Valla DC.

Budd-Chiari syndrome: a review by an expert panel. J Hepatol 2003; 38: 364-371.

2. Menon KVN, Shah V, Kamath PS. The Budd-Chiari syndrome. N Engl J Med 2004; 350: 578-85.

3. Orons PD, Zajko AB. Angiography and interventional procedures in liver transplantation. Radiol Clin North Am 1995; 33: 541-8.

4. Sze DY, Semba CP, Razavi MK, Kee ST, Dake MD. Endovascular treatment of hepatic venous outflow obstruction after piggyback technique liver transplantation. Transplantation. 1999; 68: 446-9.

5. Lee, SG, Park, KM, Hwang S, Kim K, Ahn C, Moon D, et al. Anterior segment congestion of a right liver lobe graft in living-donor liver transplantation and strategy to prevent congestion. J Hepatobiliary Pancreat Surg. 2003; 10: 16-25.

6. Tanaka T, Kiuchi T. Living-donor liver transplantation in the new decade: perspective from the twentieth to the twenty-first century. J Hepatobiliary Pancreat Surg. 2002; 9:

$218-22$

7. Cheng YF, Chen YS, Huang TL, de Villa V, Chen TY, Lee TY, et al. Interventional radiologic procedures in liver transplantation. Transplant Int 2001; 14: 223-9. 
8. Egawa H, Tanaka K, Uemoto S, Someda H, Moriyasu F, Sano K, et al. Relief of hepatic vein stenosis by balloon angioplasty after living-related donor liver transplantation. Clin Transplant 1993; 7: 306-11.

9. Lee WS, John P, McKiernan K, de Ville de Goyet J, Kelly DA. Inferior vena cava occlusion and protein enteropathy after liver transplantation in children. J Pediatr Gastroenterol Nutr 2002; 34: 413-6.

10. Karrer FM, Kam I, Brandt C. Budd-Chiari syndrome after reduced-size liver transplantation. Transplant Proc 1994; 26: 187.

11. Rerksuppaphol S, Hardikar W, Smith AL, Wilkinson JLK, Goh TH, Angus P, et al. Successful stenting for Budd-Chiari syndrome after pediatric liver transplantation: a case series and review of the literature. Pediatr Surg Int 2004; 20: 87-90.

12. Nakazawa Y, Josson JR, Walker NI, Kerlin P, Steadman C, Lynch SV, et al. Fibrous obliterative lesions of veins contribute to progressive fibrosis in chronic liver allograft rejection. Hepatology 2000; 32: 1240-7. 


\section{Figure legends}

Figure 1. Computed tomography revealed stenosis of the IVC (A) and dilatation on the caudal side of the stenosed IVC (B). Focal fluid accumulation was found around the IVC (arrows).

Figure 2. Vena cavogram demonstrated narrowing of the IVC (arrow heads) (A). The pressure of the supra-hepatic IVC was 2-4 mmHg, in contrast with $24-30 \mathrm{mmHg}$ in the infra-hepatic IVC and the HV (B).

Figure 3. Computed tomography at 1.5 months after the second laparotomy demonstrated decreased ascites and normalized diameter of the IVC. 
Figure 1.
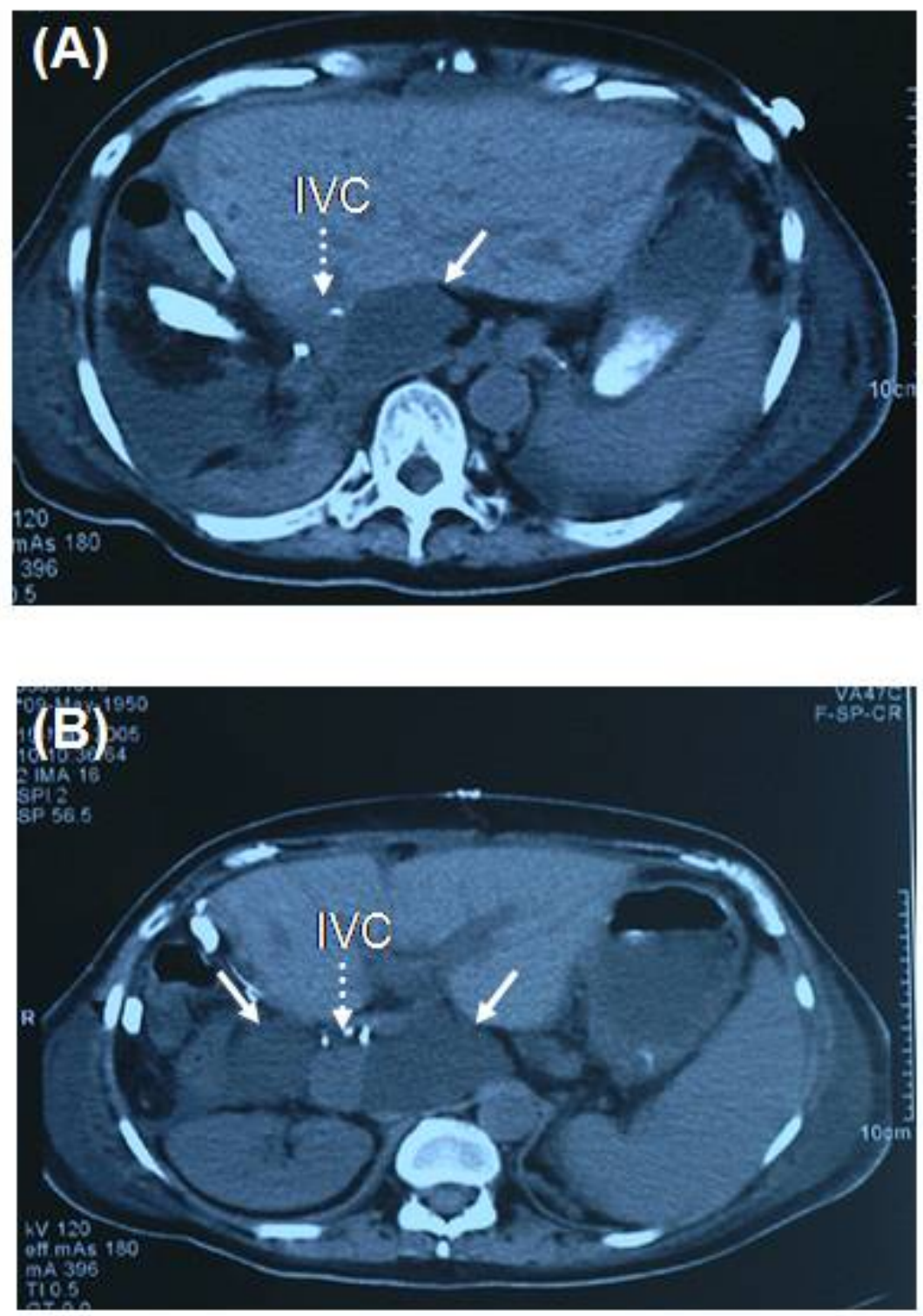
Figure 2.
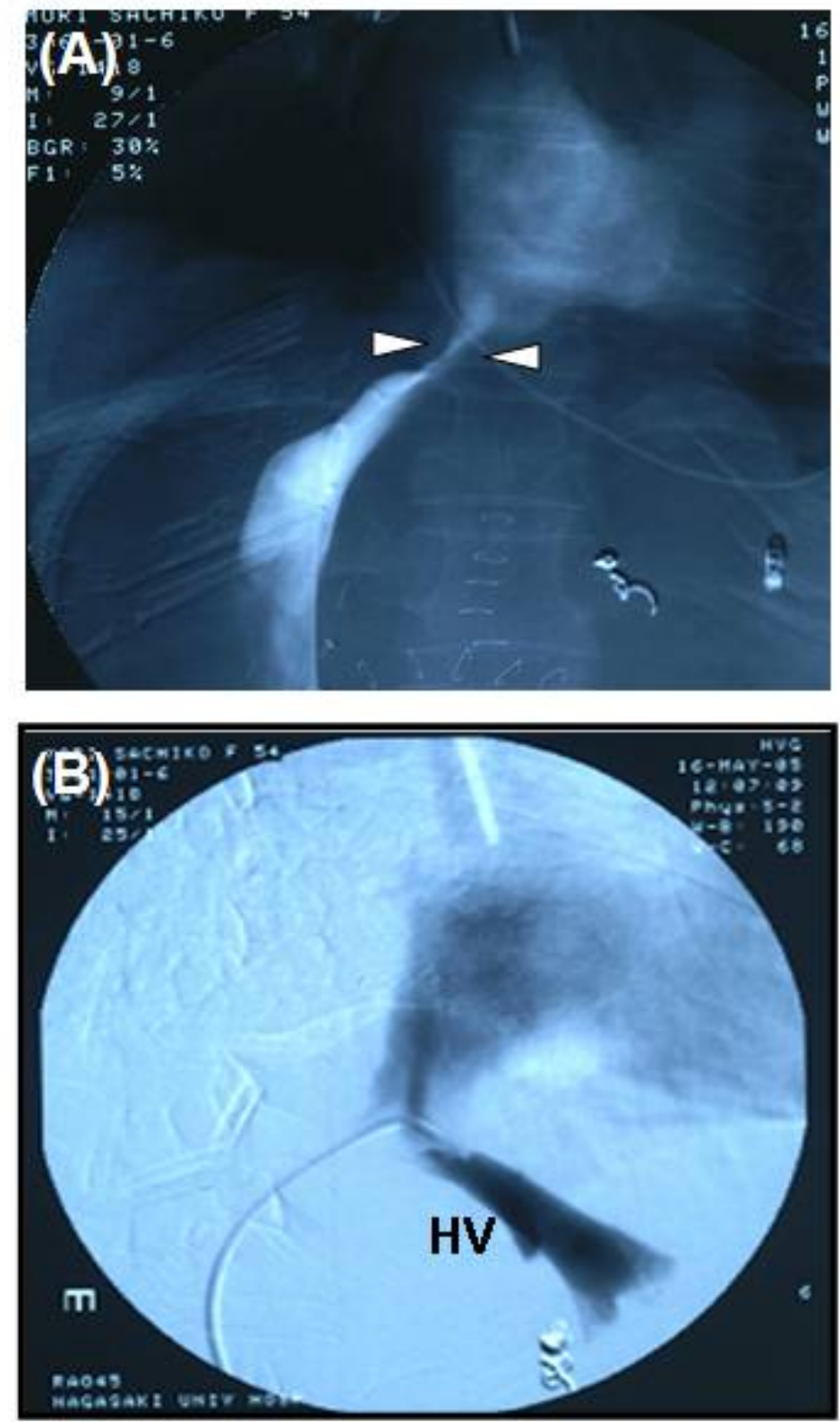
Figure 3.

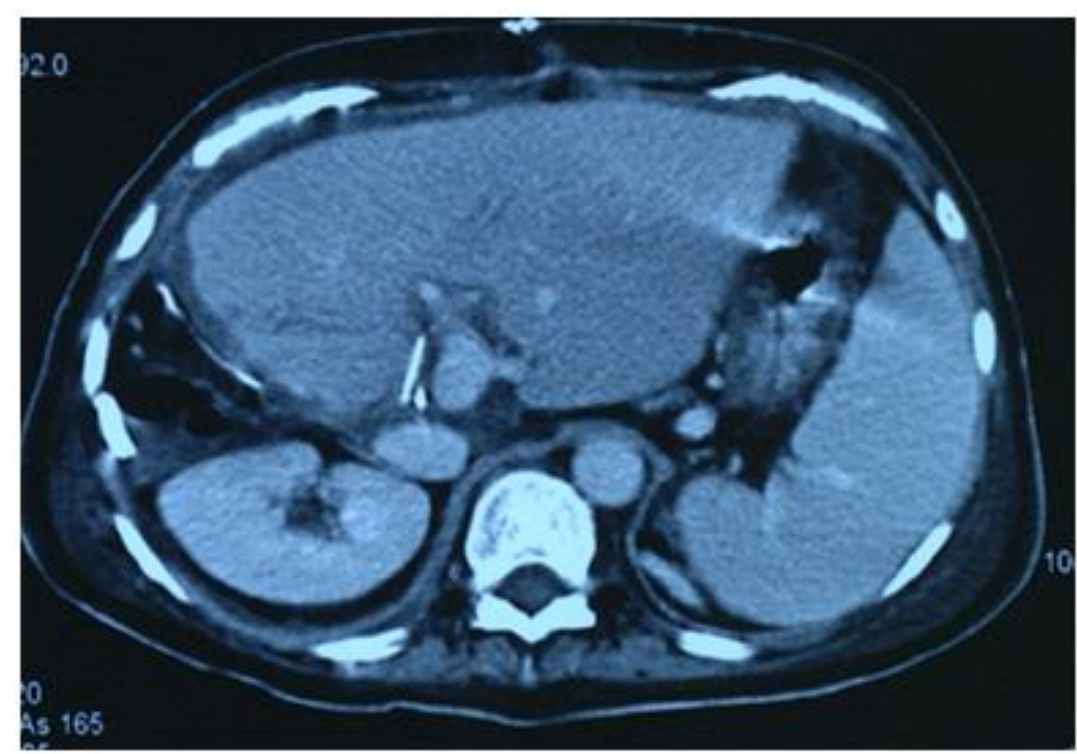

